\title{
Initial Results from Aberration Correction in STEM
}

\author{
A. R. Lupini, S. J. Pennycook, O. L. Krivanek ${ }^{*}$, N. Dellby ${ }^{*}$, P. D. Nellist* \\ Oak Ridge National Laboratory, Solid State Division, Oak Ridge TN 37831-6030 \\ *Nion Co., 1102 8th St., Kirkland, WA 98033, USA.
}

Aberration correction is an area of research with a long history in electron microscopy, dating back to the discovery by Scherzer in 1936 that the spherical and chromatic aberrations of conventional round lenses will always be positive [1]. The correction of spherical aberration $\left(\mathrm{C}_{\mathrm{s}}\right)$ is achieved by incorporating a separate aberration corrector, containing non-round elements, into the electron optical system to eliminate the $\mathrm{C}_{\mathrm{s}}$ of the round objective lens. We have recently fitted a quadrupole-octupole $\mathrm{C}_{\mathrm{s}}$-corrector [2], to the $100 \mathrm{kV}$ VG501 STEM at ORNL, shown in Fig. 1. The STEM is particularly well suited to the correction of spherical aberration because the high-angle annular dark field (HAADF) image can be directly interpreted as an image of the atomic column positions [3], is relatively insensitive to chromatic aberration and allows the simultaneous acquisition of electron energy loss spectra (EELS).

Once the spherical aberration has been eliminated, the major factors that limit the resolution of $\mathrm{a} \mathrm{C}_{\mathrm{s}}$-corrected STEM are the stabilities of the column, both electrical and mechanical, and the so-called parasitic aberrations. At ORNL, the $\mathrm{C}_{\mathrm{s}}$-corrected microscope is isolated from mechanical vibrations by means of a suspension system and sound damping surroundings. Parasitic aberrations can arise because of mis-alignments of the system, or inhomogenieties in the magnetic elements (an example is described in [4]). Accurate alignment and the flexibility to cancel any parasitic aberrations are therefore essentials in a $\mathrm{C}_{\mathrm{s}}$-corrected system. In order to achieve a higher resolution, the objective aperture size should be correspondingly increased (Fig. 2). This has the additional benefit of allowing higher current at the same probe size, thereby increasing the signal available for EELS analysis [2]. The increased aperture size also increases the precision with which the parasitic aberrations must be corrected, which requires computer control of the corrector alignment. Because conventional methods of determining the aberration function are not well suited to a STEM, instead our $\mathrm{C}_{\mathrm{s}}$-corrector uses a method based on the analysis of the electron shadow image, also known as the Ronchigram (Fig. 2) [2].

Example HAADF images of silicon "dumbbells" are shown in Fig. 3. Without $\mathrm{C}_{\mathrm{s}^{-}}$ correction, the resolution of the $100 \mathrm{kV}$ STEM is approximately $2.2 \AA$, and the dumbbells cannot be resolved. After $\mathrm{C}_{\mathrm{s}}$-correction the 2 distinct columns, separated by only $1.36 \AA$ are resolved. The correction of spherical aberration in the STEM will allow imaging and microanalysis at higher resolution than ever before.

\section{References:}

[1] O. Scherzer, Zeit. Phys 101 (1936) 593.

[2] N. Dellby et al, Journal of Electron Microscopy 50 (2001) 177.

[3] P. D. Nellist et al, Ultramicroscopy 78 (1999) 111.

[4] V. Beck, Proc. 35th EMSA (1977) 90. 


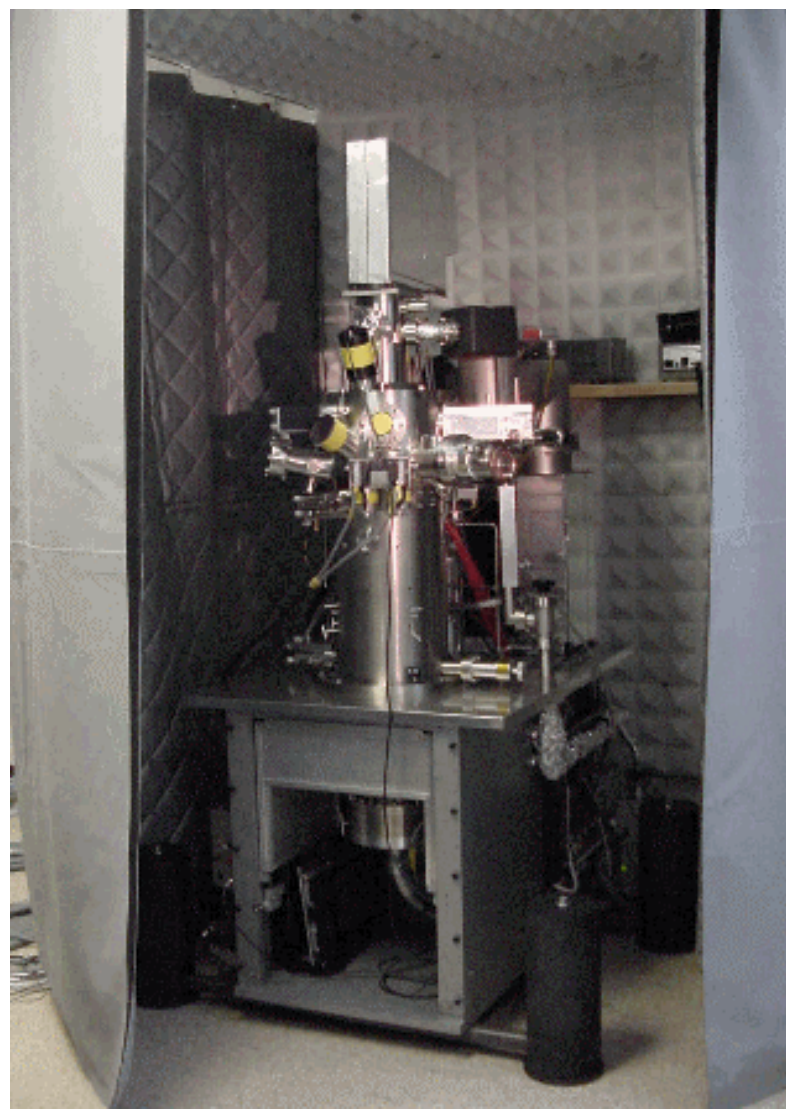

Figure 1. External view of $\mathrm{C}_{\mathrm{s}}$-corrected $100 \mathrm{kV}$ VG501 STEM at ORNL.

(a)

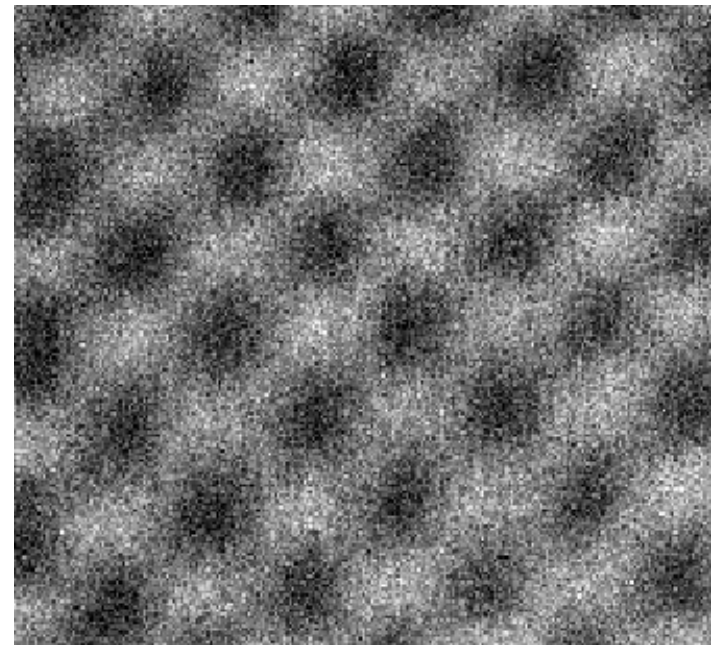

$1 \mathrm{~nm}$ (a)

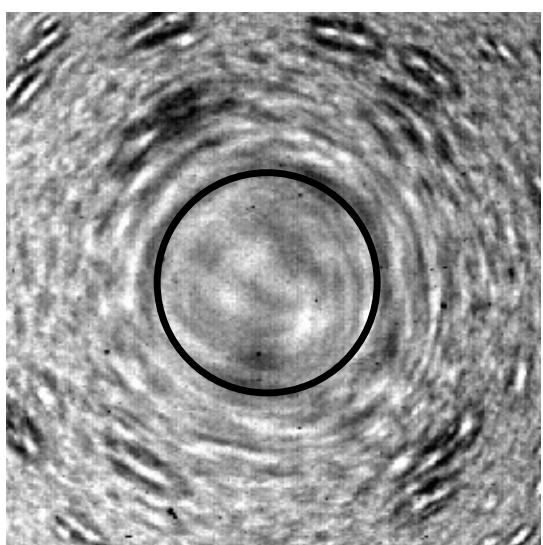

(b)

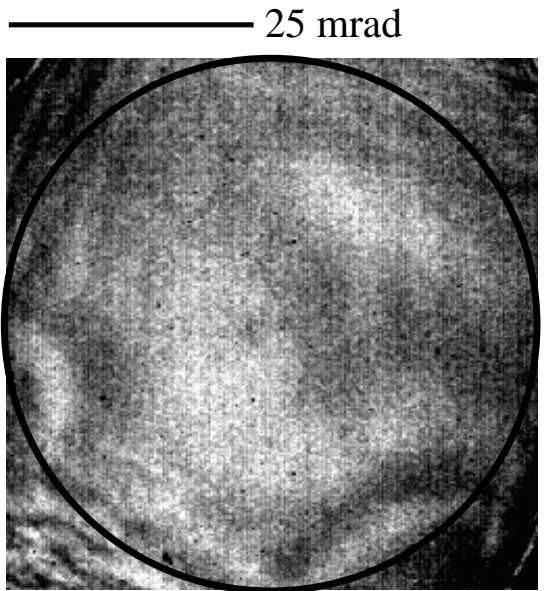

Figure 2. Ronchigrams taken near Gaussian focus (a) before and (b) after the correction of spherical aberration. In both cases the approximate size of the optimal objective aperture is indicated.

(b)

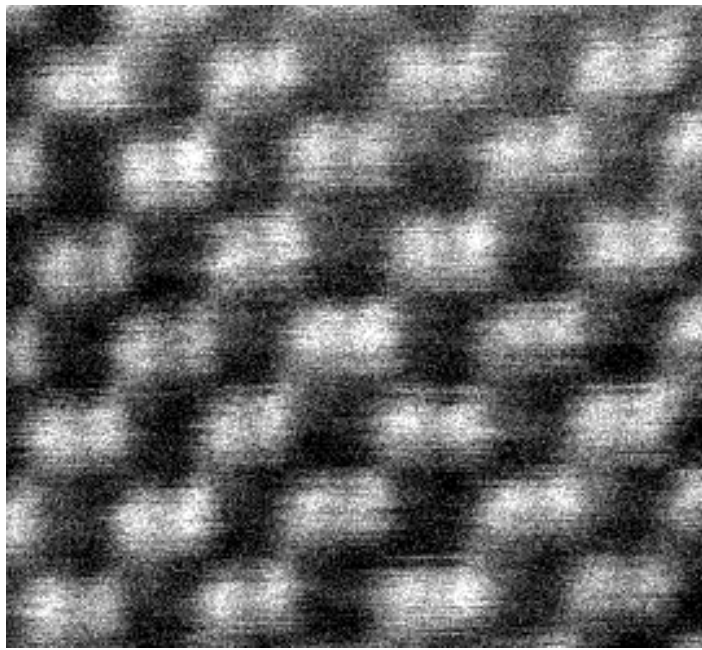

$1 \mathrm{~nm}$

Figure 3. HAADF images of silicon "dumbbells" at similar magnifications taken in a $100 \mathrm{kV}$ VG STEM (a) before and (b) after the correction of spherical aberration. 